\title{
'Lost' contact lens presenting as an intraocular foreign body
}

\author{
Stephen M Scotcher, Christopher R Canning, Ross Neville-Lamb
}

'Lost' contact lenses are often found in the conjunctival fornices. We describe a case where a lost contact lens turned up in the vitreous. To our knowledge this has not been reported previously.

\section{Case report}

A 36-year-old man sustained a perforating corneal laceration to his left eye from a piece of wire, which apparently flicked up from a pile of rubbish. He was wearing soft contact lenses at the time and the left lens was not recovered. He had no previous eye disease and was in good general health.

He was seen in eye casualty and was found to have a left visual acuity of hand movements. There was a $5.8 \mathrm{~mm}$ full thickness corneal laceration, a moderate hyphaema, a deep anterior chamber, a round pupil, and an intact crystalline lens. No intraocular foreign body was detected by plain $x$ ray.

A primary repair was performed under general anaesthesia and subconjunctival gentamicin and cefuroxime were administered (Fig 1). The following day a fibrinous anterior uveitis without a hypopyon obscured the view of his posterior segment. He received hourly drops of betamethasone and chloramphenicol, 6 hourly atropine drops $1 \%$, and intravenous cefuroxime $750 \mathrm{mg} 6$ hourly.

The uveitis improved significantly over the next 2 days but his visual acuity remained at hand movements with vitreous haemorrhage and inflammation. Prednisolone $60 \mathrm{mg}$ daily orally was started. The vitreous findings

Accepted for publication 5 September 1994

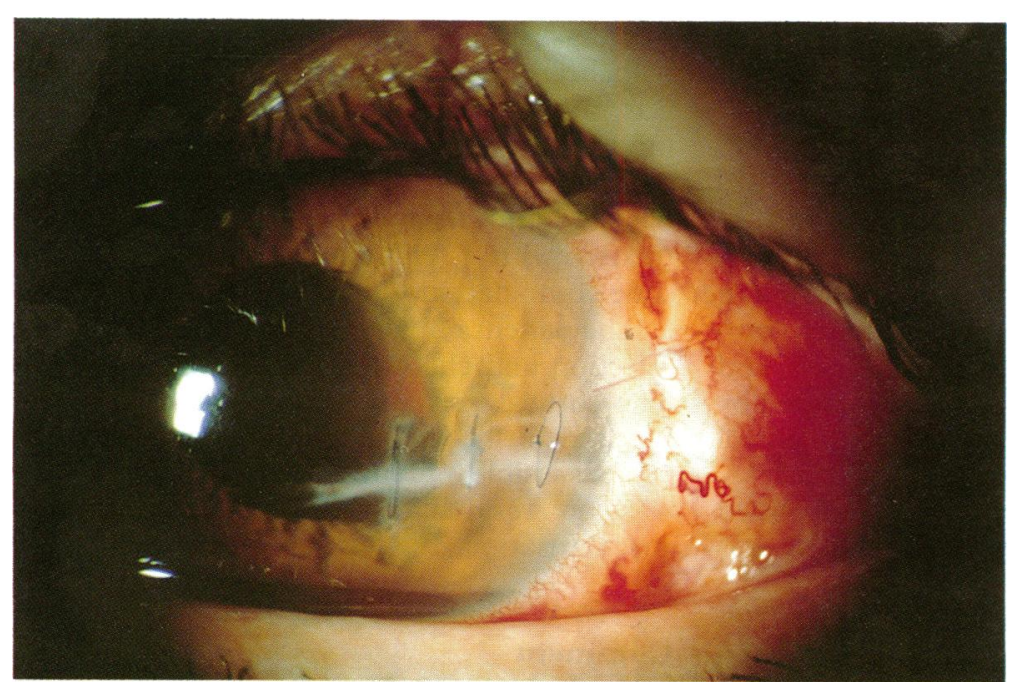

Figure 1 Anterior segment photograph showing repaired full thickness corneal laceration, left eye. In addition there is a $2 \mathrm{~mm}$ hyphaema. suggested a posterior segment injury but no iris defect or lens damage were present. A left relative afferent pupil defect developed and a $B$ scan ultrasound examination suggested retinal detachment. He was referred to the regional vitreoretinal service.

Repeat ultrasound examination showed a hyperechoic area with acoustic shadowing in the posterior segment (Fig 2). This was highly suggestive of an intraocular foreign body so an orbital computed tomography (CT) scan was performed. It was negative for foreign body.

A standard three port vitreolensectomy was carried out. During this procedure an intact soft contact lens was identified in the vitreous and removed via an enlarged sclerotomy. The vitreous contained extensive inflammatory debris and the retina was oedematous and inflamed. Postoperatively the posterior segment inflammation settled but the vision remained poor with an acuity of hand movements as a result of extensive retinal scarring.

\section{Comment}

Numerous reports exist of 'lost' contact lenses subsequently presenting as lumps in the upper lid and fornix. ${ }^{1}$ The interesting facets of this case are, firstly, that a soft contact lens can enter the vitreous via the anterior segment without any obvious damage to the iris or lens; and, secondly, that neither plain radiology nor CT scanning showed up the lens while ultrasound did.

We assume that the object which caused the corneal laceration simultaneously folded up and posted the contact lens through the corneal defect. In view of the fact that both the iris root and crystalline lens appeared intact we feel that the contact lens must have passed through the pupil and then through an unidentified zonular dehiscence to end up in the posterior segment.

Plain $x$ ray is the standard initial investigation in most centres for the detection of a suspected intraocular foreign body (IOFB). ${ }^{2}$ This method often fails to show non-metallic IOFBs. Plastic is frequently radiolucent, with the detection rate for Perspex IOFBs as low as $15 \% .^{3}$

Computed tomography offers better localisation $^{4}$ but may also miss small or non-metallic ${ }^{5}$ foreign bodies. Much has been written about the failure of CT to detect wooden foreign bodies, but there are few reports regarding CT imaging of plastic IOFBs. Plastics have a wide range of $\mathrm{CT}$ densities depending on their exact composition. Two cases of plastic IOFBs not 


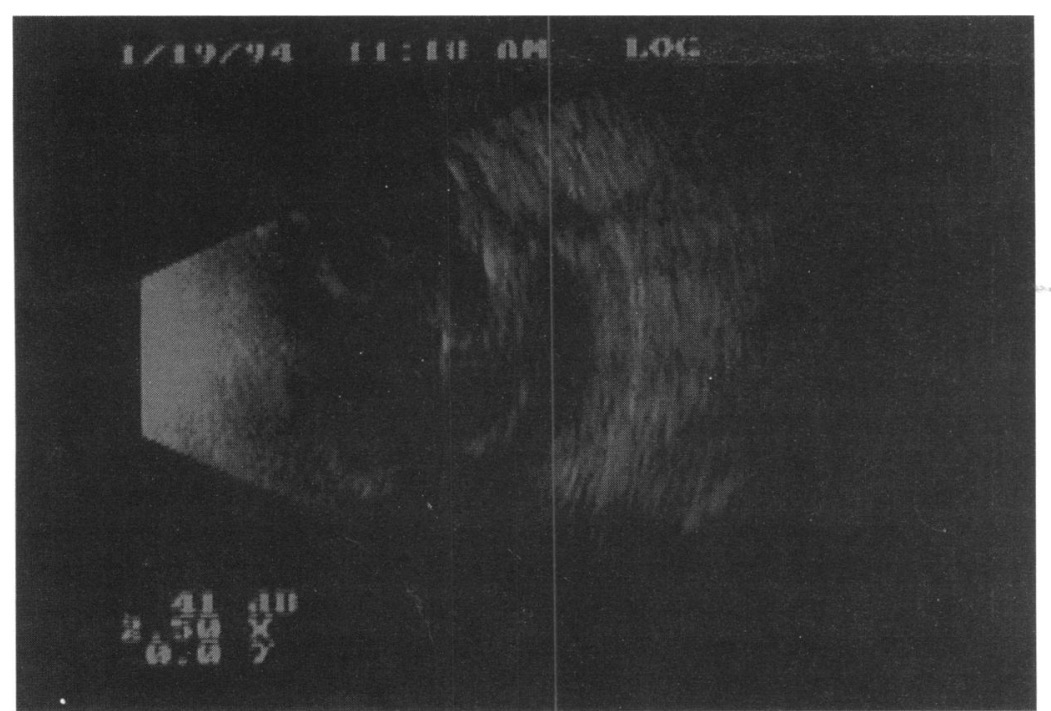

Figure 2 B scan ultrasound left eye. Note hyperechoic area with acoustic shadowing in the posterior segment suggestive of an intraocular foreign body.

correctly diagnosed by CT scan have been described. ${ }^{6}$

Ultrasonography has been advocated in view of its availability, low cost, and high detection rates. One study, ${ }^{3}$ using porcine eyes, reported an overall IOFB detection rate by ultrasound of $93 \%$ compared with $40 \%$ by plain $x$ ray. Significantly, Bryden et al's detection rate for Perspex was $96 \%$. Localisation is, of course, less accurate and the technique may not be applicable in some acutely injured eyes.

This case emphasises the need for a combined approach using plain $x$ ray, CT, and ultrasound in the complete assessment of a penetrating eye injury where an intraocular foreign body is suspected.

1 Roberts-Harry TJ, Davey CC, Jagger JD. Periocular migration of hard contact lenses. Br $₹$ Ophthalmol 1992; 76: tion of

2 Bray LC, Griffiths PG. The value of plain radiography in suspected intraocular foreign body. Eye 1991; 5: $751-4$.

3 Bryden FM, Pyott AA, Bailey M, McGhee CN. Real time ultrasound in the assessment of intraocular foreign bodies. Eye 1990; 4: 727-31.

4 Etherington RJ, Hourihan MD. Localisation of intraocular and intraorbital foreign bodies using computed tomoand intraorbital foreign bodies using

5 LoBue TD, Deutsch TA, Lobick J, Turner DA. Detection and localisation of non-metallic intraocular foreign bodies by magnetic resonance imaging. Arch Ophthalmol 1988; 106: $260-1$

6 Henrikson GC, Mafee MF, Flanders AE, Kriz RJ, Peyman GA. CT evaluation of plastic intraocular foreign bodies. Am $\mathcal{F}$ Neuroradiol 1987; 8: 378-9.

\title{
Crystalline keratopathy from Dieffenbachia plant
} sap

\author{
Benjamin Seet, Wing-Kwong Chan, Chong-Lye Ang
}

Singapore National Eye Centre, Singapore $B$ Seet W-K Chan C-L Ang

Correspondence to: Dr B Seet, Singapore National Eye Centre, 11 Third Hospital Avenue, Singapore 0316 .

Accepted for publication 5 September 1994
We report an unusual ocular injury caused by sap of the Dieffenbachia plant, in which there is acute keratoconjunctivitis associated with needle-like crystals in the cornea. Diagnosis is made from a history of contact with the sap, as well as by the presence of characteristic crystals.

\section{Case report}

A 23-year-old man was cutting bushes of Dieffenbachia (Fig 1) when sap squirted into his right eye. He complained of mild irritation which developed, within a few hours, into severe eye pain associated with redness and mild visual blurring. $\mathrm{He}$ was initially treated by his general practitioner with chloramphenicol eyedrops, and examined by us on the third day after the injury. At this time, he complained that the redness had not resolved, but he was otherwise asymptomatic.

Visual acuity in the right eye was $6 / 7 \cdot 5$. There was mild conjunctival injection and chemosis, as well as slight subconjunctival haemorrhage. The corneal epithelium was intact at the time of examination, but fine refractile needle-like blue crystals were seen within the cornea, extending from the subepithelial region to the posterior stroma. These were distributed predominantly in the interpalpebral and inferior cornea (Fig 2). There was no associated corneal oedema or cellular infiltration and no inflammatory activity within the anterior chamber. No abnormality was detected in the uninjured left eye. The eye was treated with a chloramphenicol dexamethasone combination, prescribed 4 hourly.

The patient remained asymptomatic and the injection and chemosis resolved after 1 week. The corneal crystals were noted to disappear spontaneously over subsequent weeks, with no crystals visible 3 months after the injury. There was no residual corneal scarring or neovascularisation (Fig 3).

\section{Comment}

The Dieffenbachia is a common household plant belonging to the Araceae family. It was 